\title{
„Die Gefahr steckt in der Geschwindigkeit, mit der sich die Dinge verändern“"
}

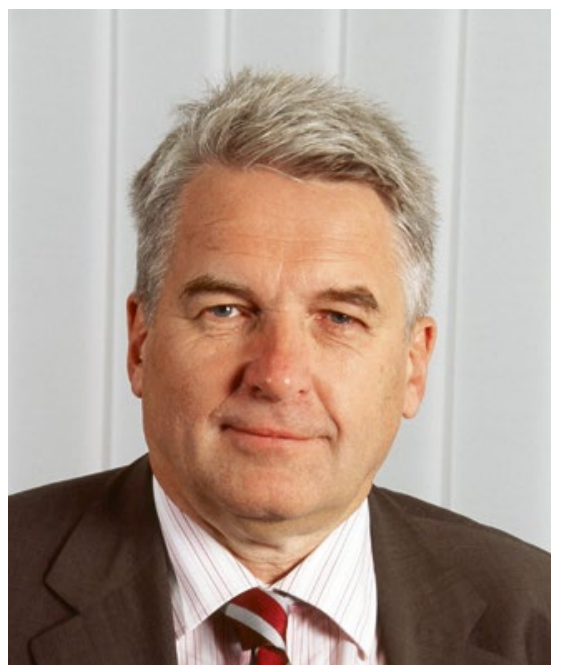

Prof. Dr. Dr. h.c. Manfred Broy ist Leiter des Instituts für Informatik an der Technischen Universität München.
ATZelektronik _ Herr Prof. Broy, mit den heutigen Methoden und Strukturen in der automobilen Softwareentwicklung bleibt das autonom fahrende Automobil eine Vision, mahnen Sie. OEMs wissen das, scheinen sich aber nicht langfristig genug vorzubereiten? BROY_Sie müssten die Neugestaltung der E/E-Architektur nicht nur lang-, sondern mittelfristig angehen, und sicher nicht allein aufgrund der Hochautomatisierung. Ich habe durchaus Verständnis für die Zwänge und Zwickmühlen der Automobilhersteller. Sie beschäftigen sich zwar mit den dringenden Zukunftsfragen. Doch wenn es zum Schwur kommt, wird das Dringliche gemacht und das Wichtigere immer wieder vertagt.

Liegen die Kunstgriffe darin, parallel zur alten Welt eine neue aufzubauen? Nein. So revolutionär ist das Ganze nicht. Es geht um eine schrittweise Migration. Der Schlüssel liegt in der Integrationsfähigkeit.

Wie groß ist der Umsetzungsdruck? Sehr hoch, weil sich sonst die Zukunftspläne der Autohersteller nur schwer oder gar nicht realisieren lassen. Denn die inhaltlichen Schwerpunkte in den kommenden zehn Jahren werden sich nochmals verlagern, hin zu einer Verdopplung der Aufwände von Software-, Informatik- und Digitalisierungsthemen. Wenn man das in den Griff bekommen will, benötigt man neue Systematiken einerseits und neue, modulare und flexible Softwarearchitekturen andererseits. Die Anbindung an die Backends müssen neu geschnitten werden, Security-Anforderungen kommen hinzu und neue Funktion für das autonome Fahren, auch wenn sich dies später als bislang spekuliert realisieren lässt. Heute wird das eher mit Rucksackmethoden gemacht.

Die Autohersteller reagieren, und bauen mehr Software-Know-how intern au. Der Stellenwert von Software bei allen OEMs steigt. Das ist eine gute Entwicklung, wobei die technischen sowie organisatorischen Ansätze einfach nicht ausreichend durchdacht werden. Denkweisen müssen sich ändern - ein regelrechter Paradigmenwechsel steht an. Dieser Tragweite müssen sich die Topmanager meiner Ansicht nach noch klar werden. Autohersteller verkaufen heute Produkte, künftig müssen sie Dienstleistungen verkaufen, und das Produkt ist Teil der Dienstleistung. Zudem tummeln sich die neuen Konkurrenten wie Google und Apple mit einer enorm hohen Geschwindigkeit nun auch im Automobilmarkt. Smartphones haben innerhalb von zehn Jahren unsere Gesellschaft verändert. Google ist gerade mal 17 Jahre alt. Wobei wir wieder bei den flexiblen Softwarearchitekturen sind. Bei Internetfirmen werden Funktionalitäten innhalb weniger Tage freigeschaltet. In den trägen Strukturen der Autoindustrie vergehen hingegen Wochen oder gar Monate ...

... weil neue Funktionen auch sicherheitskritisch und lebensgefählich sein können. Ich weiß diesbezüglich zu unterscheiden. Bedeutsam ist, dass die OEMs ja selber schneller reagieren wollen.

Nun weisen Sie auf den notwendigen Wandel gebetsmühlenartig seit zehn Jahren hin. Bleiben der Branche weitere zehn? Sicher nicht. Die Gefahr steckt in der Geschwindigkeit, mit der sich die Dinge verändern.

\section{Und das soll im Topmanagement noch} nicht angekommen sein?

Nicht mit dem Bewusstsein über die Tragweite. Die unternehmerische Kernfrage lautet doch auch, welche Wertschätzung man dem Berufsbild der Softwareentwickler entgegenbringt und mit welcher handwerklichen Sorgfalt neue Software entsteht. Schaut man Entwicklern in den USA über die Schulter, weiß man, wo die Autoindustrie noch krankt.

Herr Prof. Broy, ich bedanke mich für das Gespräch.

INTERVIEW: Markus Schöttle FOTO: TUM 